\title{
A SUBGLACIAL BOUNDARY-LAYER REGELATION MECHANISM
}

\author{
By E.M. SHOEMAKER
}

(Faculty of Science, Simon Fraser University, Burnaby, British Columbia V5A IS6, Canada)

\begin{abstract}
Heat input to basal ice at subglacial lowpressure regions, such as exist on the lee side of bed bumps including regions of ice-bed separation, is shown to melt basal ice internally in a narrow boundary layer at most centimeters thick. Before ice at the ice-bed interface can begin to melt, the heat input $Q$ must exceed a critical value $Q^{*}, Q^{*}$ increases rapidly with an increase in the difference $\Delta P$ between the nominal (global) overburden pressure and the magnitude of the (local) normal stress acting between the ice and bed or ice and water pocket. Because of the non-linear nature of the flow law, the thickness of the boundary layer decreases rapidly with increasing $\Delta P$. The ice in the boundary layer is likely to be soft with a high water content. Under certain conditions, a regelation cycle may exist between the boundary layer and the water in a subglacial cavity. The boundary layer is sufficiently narrow that the processes can reach steady state while ice traverses subglacial low-pressure regions of length the order of $0.01-$ $0.1 \mathrm{~m}$. The regelation phenomenon may preserve or aid the formation of narrow debris-rich ice layers at the base of temperate glaciers.
\end{abstract}

\section{NOMENCLATURE}

$h \quad$ Thickness of ice cover

$J \quad$ Defined by Equation (9)

$m \quad$ A melting rate defined in Equation (5)

$P_{0} \quad$ Overburden pressure equal to $\rho_{\mathrm{i}} g h$

$p_{\mathrm{w}} \quad$ Water-pocket pressure

$Q \quad$ Heat flux equal to $q(0)$

$Q^{*} \quad$ Critical value of $Q$ such that $q(0)$ is just sufficient to produce a hydrostatic state of stress on the ice-water pocket interface

$q \quad$ Heat-flux field defined in Equation (4)

$s_{x}, s_{y}, s_{z}$ Cartesian components of normal stress deviators

$x, y, z$ Cartesian coordinates with origin at ice-water interface, $y$-axis directed into ice

$B \quad$ Parameter defined near Equation (6)

$\dot{\varepsilon}_{x}, \dot{\varepsilon}_{y}, \dot{\varepsilon}_{z}$ Cartesian components of strain-rate field

$\theta^{x,} y, \varepsilon z$ Temperate field relative to temperature at icewater pocket interface defined in Equation (3)

$\theta_{\infty} \quad$ Asymptotic value of ice temperature given by $-c\left(P_{0}-p_{\mathrm{W}}\right)$

$\sigma_{x}, \sigma_{y}, \sigma_{z}$ Cartesian components of normal stress field

\section{INTRODUCTION}

It is generally accepted that small water-filled cavities in the lee of bedrock bumps are ubiquitous, at least where the ice-sliding velocity is not abnormally small (Lliboutry, 1958, 1968, 1978). Large filled or partially filled cavities beneath thick ice also are not uncommon (Schneider, 1939, 1948; La Chapelle, 1968; Paterson and Savage, 1970; Vivian and Bocquet, 1973). Normally, the water pressure $p_{w}$ in such cavities is less than the nominal (global) overburden pressure $P_{0}=\rho_{\mathrm{i}} g h$ where $\rho_{\mathrm{i}}$ is the ice density and $h$ is the ice thickness. The pressure difference $\Delta P=P_{0}-P_{\mathrm{w}}$ can be large. At least on steep bed slopes, $\Delta P$ can approach $\rho_{\mathrm{i}} g h$ (Kamb, 1970; Vivian and Bocquet, 1973). Because ice near the bed is in the presence of such a reduced pressure environment during a large part, up to one-half, of its existence before being melted, it is important to understand the effect of this environment upon properties such as the stress condition and melting process of ice near the bed.

We shall examine the steady-state stress and temperature fields in temperate ice near the roof of a subglacial water-filled cavity with the condition $\Delta P>0$ assumed to apply. From equilibrium considerations, the component of normal stress directed normal to the ice-water interface (the exterior normal stress) must be continuous across the interface. However, the component of normal stress directed tangentially to the ice-water interface (the interior normal stress) need not be equal to the exterior normal stress at the interface. This should be the case if $\Delta P>0$, since the magnitude of the interior normal stress could be expected to approach the overburden pressure. Similar conditions exist on the lee sides of bedrock bumps, whether or not ice-bed separation occurs.

Consider a crystal or grain situated directly above an ice-water pocket interface (Fig. 1) under conditions where heat input from the water pocket causes melting. Assume that $\Delta P>0$ with $\left|\sigma_{x}\right|=\left|\sigma_{z}\right|>\left|\sigma_{y}\right|=p_{w}$ at the
interface, $y=0$. Then, according to the conventional Gibbs-Kamb model (Kamb, 1961; McLellan, 1966), melting occurs on parts of grain boundaries with the highest magnitude of normal stress. In the present case, this will be one or more of the lateral crystal faces A, B, C, ... of

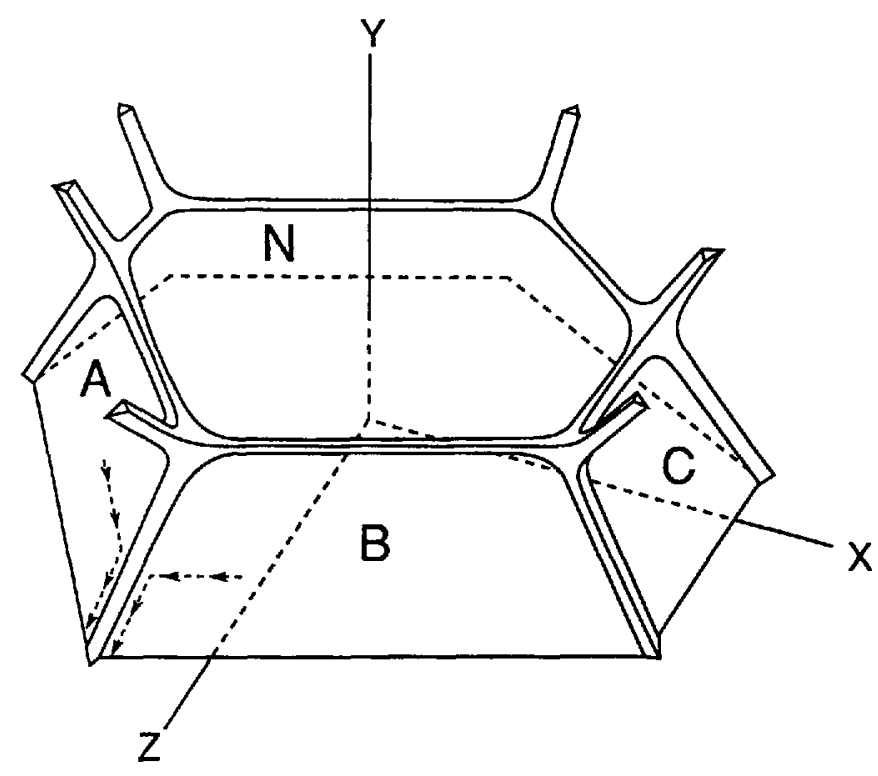

Fig. 1. The vein-node system (modified from Nye. 1989; not to scale.) Dotted arrows indicate drainage paths. Base of grain is on ice-water pocket interface. 
Figure 1; melting will not occur on face $\mathrm{N}$ or the ice-water pocket interface unless or until the magnitude of normal stress on either of these faces equals or exceeds the magnitude of normal stresses on the lateral faces. Thus, melting will occur preferentially at interior grain boundaries rather than on the ice-water pocket interface.

The phenomenon just outlined was recognized by Nye and Mae (1972), who also of fered experimental confirmation of the formation of water lenses at interior grain boundaries. Further relevant work was done by Lliboutry (1971, 1976, 1987, p. 160-64), Nye and Frank (1973), and Nye $(1976,1989)$.

The consequences of melting interior to the ice bordering a water pocket or other low-pressure regions will be examined. It will be shown that interior melting and consequent stress redistribution is confined to a narrow boundary layer of ice, no more than centimeters thick. It is expected that the water content in the boundary-layer ice is high.

It is assumed that the characteristic length of the water pocket is large compared to ice-crystal size and boundarylayer thickness. Thus, plane-strain conditions apply and the half-space geometry of the problem examined in the next section is justified if these relative size restraints are satisfied.

Because of the grain structure, the melting process is highly non-homogeneous. In addition, conduction across grain boundaries involves different coefficients than conduction interior to grains. To illustrate qualtitative effects, it is sufficient and convenient to replace the discrete grain system by a homogeneous isotropic system where conduction, melting, and water drainage take place uniformly on any plane parallel to the ice-water pocket interface. Certain characteristics of the grain structure, however, will be incorporated in the model.

\section{ANALYSIS}

Consider the steady-state situation of half space $y>0$ occupied by temperate ice throughout. Water occupies $y<0$ at temperature $\theta_{\mathrm{w}}$ and pressure $p_{\mathrm{w}}$. Assume that heat is released by the water to the ice at a uniform rate per unit area $Q \geqslant 0$. (The source of heat input will be discussed later.) If $Q=0$, the system is in thermodynamic equilibrium and the uniform equilibrium stress field in the ice is taken to be

$$
\sigma_{x}^{0}=\sigma_{z}^{0}=-P_{0}, \quad \sigma_{y}=-p_{\mathrm{w}}, \quad y \geqslant 0
$$

where $P_{0} \geqslant p_{\mathrm{w}}$ and $\sigma_{x}, \sigma_{y}, \sigma_{z}$ are the $x y z$ components of normal stress. The usual sign convention (tensile stresses are positive) is observed for stresses. Gravitational forces are neglected. We shall refer to the state corresponding to $Q=0$ as the ground state.

In the more general case where $Q>0$, the stress field is

$$
\sigma_{x}=\sigma_{z}=-P_{0}+\Delta \sigma(y), \quad \sigma_{y}=-p_{w}, \quad y \geqslant 0
$$

where $\Delta \sigma(y) \geqslant 0$ remains to be determined. The stress redistribution resulting in $\Delta \sigma(y)$ originates from internal melting of ice in a boundary layer adjoining the ice-water pocket interface as will be shown.

The Clausius-Clapeyron equation (Paterson, 1981, p. 192), which is known to apply in the hydrostatic stress case, is assumed to apply in the non-hydrostatic stress case. Neglecting non-homogeneities due to the grain structure, the equilibrium temperature can be verified to be

$$
\theta(y)=-c\left(P_{0}-p_{\mathrm{W}}-\Delta \sigma(y)\right)
$$

where $c=9.8 \times 10^{-8} \mathrm{~K} \mathrm{~Pa}^{-1}$ for air-saturated water (Paterson, 1981, p. 193). $\theta$ has arbitrarily been set to zero when $P_{0}-p_{\mathrm{w}}-\Delta \sigma(y)=0$ which renders $\theta$ the temperature relative to that at the ice-water pocket interface. Equation (3) is consistent with the Gibbs-Kamb model (Kamb, 1961) which states that, if higher-order terms are neglected, the melting point is determined by the normal stress on the interface where melting occurs. Therefore, if melting occurs somewhere on the boundary of a grain which is at a uniform temperature, it can only occur on those segments of the grain boundary where the magnitude of the normal stress is maximized. (The fact that melting occurs preferentially on the highest stressed regions of a grain boundary causes the temperature in the grain to be non-uniform with heat flowing towards the regions where melting occurs.) In the present case, the maximum. normal stress magnitude occurs on planes parallel to the $y$-axis and is $P_{0}-\Delta \sigma(y)$ where it is assumed, to be verified, that $P_{0}-\Delta \sigma(y)>p_{\mathrm{w}}$. If this inequality is satisfied, melting will occur exclusively on grain boundaries parallel, or nearest parallel, to the $y$-axis. (This is the assumption leading to Equation (3).) Melting cannot occur on the ice-water pocket interface $y=0$ unless the inequality $P_{0}-\Delta \sigma(0) \leqslant p_{\mathrm{w}}$ is satisfied. These conclusions are in accordance with the experiments of Nye and Mae (1972).

Melting at interior grain boundaries produces a stress redistribution so that $\Delta \sigma(y)$ is not identically zero. There are two general cases: I. $Q$ is sufficiently large that melting occurs on the ice-water pocket interface and the stress state there is necessarily hydrostatic with $P_{0}-\Delta \sigma(0)=p_{\mathrm{w}}$; II. $Q$ is insufficiently large to cause melting on the ice-water pocket interface and $P_{0}-\Delta \sigma(0)>p_{\mathrm{w}}$. We will initially focus on the special version of case $I$ where $Q=Q^{*}$ is just sufficient to cause melting on the ice-water pocket interface.

The heat balance in the ice includes conduction, heat absorption by melting at grain boundaries, strain-energy release, and the heating of melt water generated at $y>0$ as it drains along the positive temperature gradient to the water pocket at $y=0$. The latter two contributions may be neglected. While the contribution of water drainage to heat balance may be neglected, intergranular water drainage (or the lack of it) can influence the stress field; the effect of water drainage will be addressed later.

Steady-state heat conduction for the homogeneous isotropic model is described by

$$
q=-k \mathrm{~d} \theta / \mathrm{d} y
$$

where $q$ is the heat flux and $k$ is the conductivity, assumed to be constant.

Conversion of ice to water obeys

$$
\mathrm{d} q / \mathrm{d} y+L_{\mathrm{f}} \rho_{\mathrm{i}} m(y)=0
$$

where $L_{\mathrm{f}}$ is the heat of fusion, $\rho_{\mathrm{i}}$ is the ice density, and $m$ is the net interior melting rate per unit volume. In the derivation of Equation (5), it is assumed that any refreezing at level $y$ is of melt water generated by melting at the same $y$ value.

As noted, melting occurs on crystal faces most nearly parallel to the $y$-axis. Thus, interior melting on $y>0$ would act to reduce the dimensions of the ice in the $x$ and $z$-directions if it were not for the fact that the constraint of plane strain implies that the displacement of ice in the $x$ - and $z$-directions must be independent of $y$. Thus, to balance interior melting, the ice undergoes a tensile strain-rate in the $x$ - and $z$-directions due to the components of tensile stress $\sigma_{x}=\sigma_{z}=\Delta \sigma(y)$ appearing in Equation (2)

The strain-rate field is affected by the proportion of the internally generated melt water which drains to the water pocket. Following the general outline of Nye and Mae (1972), we consider two limiting cases for the disposition of melt water formed on the lateral faces A, B, C, ... of Figure 1. At one extreme there is no drainage of melt water to the water pocket. Melt water remains on the grain faces in the form of water lenses and/or is transported to and stored in the veins. Thus, as internal melting proceeds, the quantity of stored water increases. At the other extreme, the melt water is evacuated to the water pocket at the rate it is formed. (This does not negate water storage but does imply a steady-state water content in the ice.) Evacuation is by way of the veins (Fig. 1) which are at a pressure somewhere between $P_{0}-\Delta \sigma(y)$ and $p_{\mathrm{w}}$ (Nye and Mae, 
1972). The negative pressure gradient required for vein flow is provided by the gradient in $\Delta \sigma(y)$ which can be very large, as will be shown by calculation.

It is impossible to establish the proportion of melt water which will be evacuated. Lliboutry (1971) felt that veins are blocked by ice flow. However, mechanical vein blockage is most likely to occur where shear strains are high, near the bed where the basal shear stress is high. I do not believe that mechanical blockage is likely near the roof of a cavity where the shear stress vanishes. Lliboutry (1971) also pointed out that the concentration of salts and air bubbles, which occurs mainly in the veins, also reduces the mobility of water in the lens-vein system.

The proportion of melt water which is evacuated may also be affected by the time interval for drainage to become established. The time interval for the experiments of Nye and Mae was the order of an hour, whereas the time required for ice to slide over a water pocket is at least a day. Over the larger time period, vein drainage is more likely to be achieved, particularly in view of the potentially large negative pressure gradient associated with $\Delta \sigma(y)$ acting over a boundary layer only centimeters thick.

Since the actual state of drainage must lie between the two extremes of no drainage and perfect drainage, results for these extreme cases should place bounds upon the actual behaviour of the system.

Assume that all melting takes place on crystal faces which are strictly parallel to the $y$-axis. Assume that any increases in water stored in veins or at crystal faces at level $y$ comes from melt water formed at the same level. Let $B$ be the fraction of melt water stored and $(1-B)$ the fraction evacuated to the water pocket, and assume that $\beta$ is independent of $y$.

If no additional strain-rates were imposed in the $x$ and $z$-directions in response to ice melting, a compressive strain-rate normal to the $y$-axis of amount $\left(1-\beta \rho_{\mathrm{i}} / \rho_{\mathrm{w}}\right) m / 2$ would exist, where $\rho_{w}, \rho_{i}$ are the densities of water and ice, respectively. In order to maintain the plane-strain configuration, this shrinkage must be negated so that a strain-rate field, associated with the stress field of Equation (2) through a flow law, exists, given by

$$
\dot{\varepsilon}_{x}=\dot{\varepsilon}_{z}=\dot{\varepsilon}_{x}^{0}+\left(1-\beta \rho_{\mathrm{i}} / \rho_{\mathrm{W}}\right) m / 2
$$

where $\dot{\varepsilon}_{x}^{0}$ is the ground-state strain-rate. We shall not be concerned with the strain-rate component $\dot{\varepsilon}_{y}$.

We assume that ice deformation is entirely due to secondary creep and apply a flow law of the tensorial form

$$
\dot{\varepsilon}_{i j}=D_{0} \omega(J) s_{i j} / \sigma_{0}
$$

where

$$
\begin{aligned}
\omega(J) & =0.356+0.342 J+0.0317 J^{2} \\
J & =\left(s_{x}^{2}+s_{y}^{2}+s_{z}^{2}\right) / 2 \sigma_{0}^{2}
\end{aligned}
$$

and $\sigma_{0}=10^{5} \mathrm{~Pa}, D_{0}=3.171 \times 10^{-8} \mathrm{~s}^{-1}$ (Smith and Morland, 1981). Here, $s_{x}, s_{y}, s_{z}$ are the stress deviators corresponding to $\sigma_{x}, \sigma_{y}, \sigma_{z}$, respectively. $J$ is a scaled version of the second invariant of the stress-deviator tensor (Paterson, 1981 , p. 31). Equations (7) and (8) represent a polynomial fit to Glen's (1955) unixial compression data. The model avoids the disadvantage of the Glen law (Paterson, 1981, p. 26) which exhibits infinite viscosity at zero stress. The relations between deviatoric normal stresses $s_{\mathrm{i}}$ and stresses $\sigma_{\mathrm{i}}$ for the present case are

$$
\begin{gathered}
s_{x}=s_{z}=\left(-P_{0}+p_{\mathrm{w}}+\Delta \sigma(y)\right) / 3, \\
s_{y}=2\left(P_{0}-p_{\mathrm{w}}-\Delta \sigma(y)\right) / 3 .
\end{gathered}
$$

From Equations (7) and (10), the ground-state strain-rate $\dot{\varepsilon}_{x}^{0}$ is

$$
\dot{\varepsilon}_{x}^{0}=D_{0} \omega\left(J_{0}\right)\left(-P_{0}+p_{w}\right) / 3 \sigma_{0}
$$

where

$$
J_{0}=\left(P_{0}-p_{w}\right)^{2} / 3 \sigma_{0}^{2}
$$

from Equations (9) and (10).

For the general case, when $Q>0$, we may solve for $m(y)$ from Equation (6) with $\dot{\varepsilon}_{x}$ determined from Equations (7) and (10). Thus,

$$
\left[1-\frac{\beta p_{\mathrm{i}}}{P_{\mathrm{w}}}\right] \frac{m}{2}=-\dot{\varepsilon}_{x}^{0}+\frac{D_{0} \omega(J)}{3 \sigma_{0}}\left(-P_{0}+p_{\mathrm{w}}+\Delta \sigma(y)\right) .
$$

For the particular case under consideration where $Q=Q^{*}$, the system of Equations (3), (4), (5), and (13) may be solved numerically for $\theta, q, \Delta \sigma$, and $m$ subject to the boundary condition

$$
\theta(0)=0
$$

or, equivalently, $\Delta \sigma(0)=P_{0}-p_{\mathrm{w}}$ as follows from Equation (3). (The stress state is hydrostatic at the ice-water pocket interface.) A second boundary condition may be expressed in several equivalent ways as

$$
\begin{gathered}
\theta(y) \rightarrow-c\left(P_{0}-p_{\mathrm{w}}\right), \quad y \rightarrow \infty \\
(\Delta \sigma(y) \rightarrow 0, \quad q(y) \rightarrow 0, \text { or } m(y) \rightarrow 0 \text { as } y \rightarrow \infty) .
\end{gathered}
$$

Note that $Q^{*}=q(0)$ is determined as part of the solution.

$P_{0}-p_{\mathrm{w}}$ is a forcing parameter. If $P_{0}-p_{\mathrm{w}}=0$, then $Q^{*}=0$ and the stress field reduces to the ground state which, in this case, is the uniform hydrostatic field $\sigma_{x}=\sigma_{y}=\sigma_{z}=-p_{w^{*}}$

In case $Q>Q^{*}$, the excess heat $Q-Q^{*}$ goes into melting ice at the ice-water pocket interface. Otherwise, the previous formulation and results remain intact corresponding to the heat part $Q^{*}$ which melts ice internally on $y>0$.

The previous formulation must be amended when applied to the case $Q<Q^{*}$. Boundary condition (14) must be replaced by

$$
q(0)=-k \frac{\mathrm{d} \theta}{\mathrm{d} y}(0)=Q .
$$

$\theta(0)<0$ is now obtained as part of the solution. The stress state on the ice-water pocket interface is non-hydrostatic.

\section{RESULTS AND INTERPRETATION}

A fully numerical solution is required for the general non-linear case. However, the asymptotic case for $\Delta P=P_{0}-p_{\mathrm{w}} \rightarrow 0$ results in a linear equation. Insight is gained from this solution.

For $\Delta P$ small $\omega(J) \rightarrow 0.356$ in Equation (8) and $\dot{\varepsilon}_{x}^{0} \rightarrow$ $0.356 / 3 \sigma_{0}\left(D_{0}\left(-P_{0}+p_{w}\right)\right)$ in Equation (11). Making these substitutions, the differential equation in $q$, for example, becomes

$$
\frac{\mathrm{d}^{2} q}{\mathrm{~d}_{y}{ }^{2}}-r^{2} q=0
$$

where

$$
r^{2}=0.712 L_{\mathrm{f}} \rho_{\mathrm{i}} D_{0} / 3 \sigma_{0} k c\left(1-\beta \rho_{\mathrm{i}} / \rho_{\mathrm{w}}\right) .
$$

The solution to the boundary-value problem is

$$
q=c\left(P_{0}-p_{\mathrm{w}}\right) k r \exp (-r y)
$$

so that

$$
Q^{*}=c\left(P_{0}-p_{\mathrm{W}}\right) k r
$$

Similarly, the solution for $\theta$ is

$$
\theta=c\left(P_{0}-p_{\mathrm{w}}\right)[\exp (-r y)-1] .
$$

The characteristic thickness of the boundary layer, the scale depth $y^{*}$ at which the exponential solutions decay by the multiplicative factor $1-1 / \mathrm{e}$, is seen to be

$$
y^{*}=1 / r
$$

for $\Delta P$ small. Therefore, by calculation, if $\beta=0$ (perfect drainage), $y^{*}=95 \mathrm{~mm}$; if $\beta=1$ (no drainage), $y^{*}=$ 
$27 \mathrm{~mm}$. It will be seen that for the non-linear model $y^{*}$ decreases as $\triangle P$ increases. Thus, the characteristic scale thickness of the boundary layer is bounded above by $95 \mathrm{~mm}$. Subsequent results will be confined to the case $\beta=0$.

Because of internal melting, the boundary layer is apt to have a high water content. On the other hand, Equation (8) applies to ordinary glacier ice. It is known that the viscosity of ice at moderate stress levels decreases with increasing water content up to at least an order of magnitude (Duval, 1977). However, the effect at low stress levels is not known. If we assume that, for $\triangle P$ small, the coefficient 0.356 in Equation (8) is increased to 3.56, $r^{2}$ values in Equation (18) are increased by a factor of 10 and $y^{*}$ values are decreased by a factor of $1 /(10)^{\frac{1}{2}}$.

Figure 2 graphs $\theta / \theta_{\infty}$ versus $y$ for the case $Q=Q^{*}$ and $\beta=0$ with $P_{0}-p_{\mathrm{w}}$ as a parameter. Here, $\theta_{\infty}=-c\left(P_{0}\right.$ $\left.-p_{w}\right)$, from Equation (3), is the asymptotic value of the melting temperature as $y \rightarrow \infty$. Since $c \Delta \sigma(y) / \theta_{\infty}=\theta / \theta_{\infty}-1$, from Equation (3), Figure 2 can also be interpreted as the variation in dimensionless stress $c \Delta \sigma(y) / \theta_{\infty}$. The exponential nature of $\theta(y)$ is apparent. It is also clear that the width of the boundary layer where the variables $\theta, q, m$, and $\Delta \sigma$ are changing rapidly is generally very small but increases rapidly with decreasing $P_{0}-p_{\mathrm{w}}$.

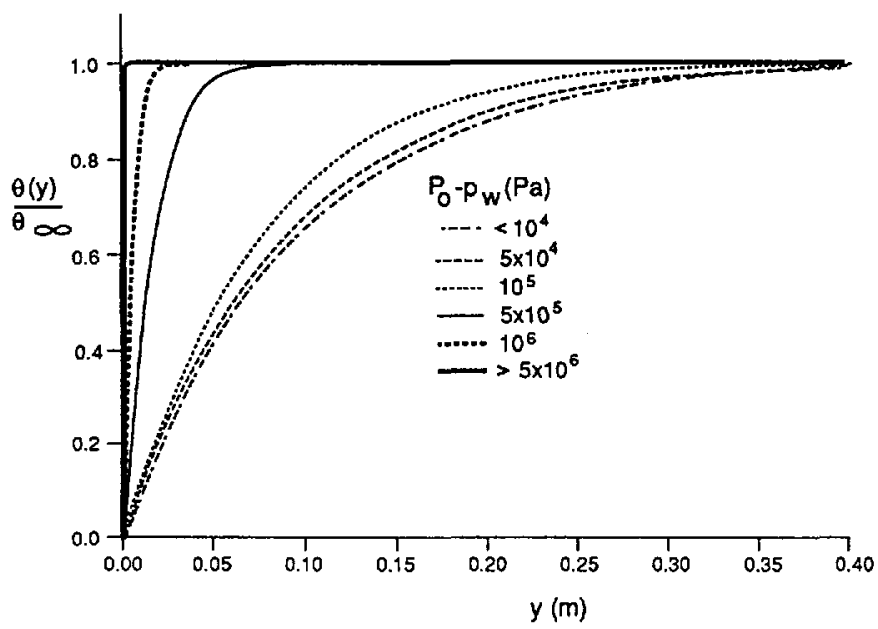

Fig. 2. $\theta(y) / \theta_{\infty}$ for various values of $P_{0}-p_{w}$ and $Q=Q^{*}$ assuming perfect drainage.

Table I shows the rapid growth of $Q^{*}=q(0)$ with increasing $P_{0}-p_{w}$ for the case $\beta=0$. The standard geothermal constant is $0.05 \mathrm{~J} \mathrm{~m}^{-2} \mathrm{~s}^{-1}$ and basal heat input might be expected to lie in the range $0.05-1 \mathrm{~J} \mathrm{~m}^{-2} \mathrm{~s}^{-1}$. It follows from Table $I$ that if $P_{0}-p_{\mathrm{w}}$ exceeds, say, $5 \times 10^{5} \mathrm{~Pa}$ the inequality $Q<Q^{*}$ should be satisfied and all basal melting will be confined to the interior of the boundary layer.

Table I also shows the characteristic thickness of the boundary layer, the scale depth $y^{*}$ at which $1-\theta\left(y^{*}\right) / \theta_{\infty}=$ $1 /$ e. It is clear that both $Q^{*}$ and $1 / y^{*}$ vary in roughly the same way with $P_{0}-p_{\mathrm{w}}$. Furthermore, the boundary-layer thickness can be much smaller than typical crystal- or grain-size diameters. Reference to the $y^{*}$ values, and comparison of $Q^{*}$ values with corresponding values calculated from Equation (20), shows that non-linearities in the system begin to take effect for $P_{0}-p_{\mathrm{W}}$ between $10^{4}$ and $5 \times 10^{4} \mathrm{~Pa}$ and increase rapidly above $P_{0}-p_{\mathrm{W}}=$ $10^{5} \mathrm{~Pa}$. This non-linear behaviour is also seen in Figure 2 .

It is easy to show that, if the non-linear flow law (7) is replaced by a Newtonian flow law, $y^{*}$ is proportional to $n^{\frac{1}{2}}$ where $n$ is the viscosity. For example, taking $n=$ $10^{13} \mathrm{Pas}$ gives $y^{*} \simeq 88 \mathrm{~mm}$. This result is independent of $\Delta P$ so that the strong dependence of $y^{*}$ upon $\Delta P$ for the present model can be attributed to the non-linear flow law (7).

(For the case $Q<Q^{*}$, the temperature of the ice at the roof of the cavity, $\theta(0)$, is below the temperature of the water in the cavity, which has arbitrarily been set to zero degrees. The variation in temperature $\theta(0)$ as a function of $q(0)$ and $P_{0}-p_{\mathrm{w}}$ is shown in Table II. The values of $q(0)$ are chosen so that the initial rows in Table
TABLE I. VARIATION IN $\theta_{\infty}, Q^{*}$, AND $y^{*}$ WITH $P_{0}-p_{\mathrm{w}}$ FOR $\beta=0$

\begin{tabular}{cccc}
$P_{0}-p_{\mathrm{w}}$ & $\theta_{\infty}$ & \multicolumn{1}{c}{$Q^{*}$} & $y^{*}$ \\
$\mathrm{~Pa}$ & $\mathrm{~K}$ & $\mathrm{~J} \mathrm{~m}^{-2} \mathrm{~s}^{-1}$ & $\mathrm{~mm}$ \\
$10^{3}$ & $-9.8 \times 10^{-5}$ & $0.022 \times 10^{-2}$ & 95 \\
$5 \times 10^{3}$ & $-4.9 \times 10^{-4}$ & $0.01 \mathrm{1}$ & 95 \\
$10^{4}$ & $-9.8 \times 10^{-4}$ & 0.022 & 95 \\
$5 \times 10^{4}$ & $-4.9 \times 10^{-3}$ & 0.11 & 88 \\
$10^{5}$ & $-9.8 \times 10^{-3}$ & 0.27 & 68 \\
$5 \times 10^{5}$ & -0.049 & 5.2 & 17 \\
$10^{6}$ & -0.098 & 32 & 5.6 \\
$5 \times 10^{6}$ & -0.49 & $3.5 \times 10^{3}$ & 0.25 \\
$10^{7}$ & -0.98 & $2.8 \times 10^{4}$ & 0.065
\end{tabular}

TABLE II. VARIATION IN $\theta(0)$ AND $\triangle \sigma(0)$ WITH $P_{0}-p_{\mathrm{W}}$ AND $q(0)$. FOR CASE $Q<Q^{*}$ AND $\beta=0$

$$
P_{0}-p_{\mathrm{w}}
$$

$\theta(0)$

$\Delta \sigma(0)$

$\mathrm{J} \mathrm{m}^{-2} \mathrm{~s}^{-1}$

$\mathrm{Pa}$

K

$\mathrm{Pa}$

0.02166

$10^{4}$
$5 \times 10^{4}$
$10^{5}$
$10^{6}$
$10^{7}$

0

$-0.00401$

$-0.00912$

$10^{4}$

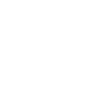

0.1144

$-0.980$

$9.1 \times 10^{3}$

$6.9 \times 10^{3}$

$\sim 0$

$5 \times 10^{5}$
$10^{6}$

$10^{5}$
$10^{6}$
$10^{7}$

0

$-0.00601$

$-0.0980$

$5 \times 10^{4}$

$3.9 \times 10^{4}$

$\sim 0$

10

$-0.980$

0.2744

$50^{5}$
$\times 10^{5}$
$10^{6}$
$10^{7}$

0

$-0.0473$

$-0.0975$

$10^{6}$

$1.7 \times 10^{4}$

$5.1 \times 10^{3}$

5.216

$5 \times 10^{5}$
$10^{6}$
$10^{7}$

$\begin{array}{ll}\times 10^{5} & 0 \\ 10^{6} & -0.0878\end{array}$

$-0.980$

$5 \times 10^{5}$

$1.0 \times 10^{5}$

$\sim 0$

31.60

$$
\begin{aligned}
& 10^{6} \\
& \times 10^{7}
\end{aligned}
$$

$-0.487$

$-0.979$

$10^{6}$

$3.1 \times 10^{4}$

$\sim 0$

II, corresponding to any particular value of $q(0)$, satisfy the condition $q(0)=Q^{*} ;$ in addition, these initial rows are shown in Table I. Thus, Table II is an extension of part of Table I to the state $Q<Q^{*}$.

Table II also shows the quantity $\Delta \sigma(0)$, calculated from Equation (3), which is a measure of the maximum stress change caused by melting at the ice-water pocket interface. The results of Table II show that non-linear effects are concentrated in a narrow range of heat input as $q(0) \rightarrow Q^{*}$.

Tables I and II results confirm that the gradient $(-\mathrm{d} \Delta \sigma(y) / \mathrm{d} y)$ can be large in the narrow boundary layer. For example, if $Q=Q^{*}$ and $P_{0}-p_{\mathrm{w}}=10^{\mathrm{E}} \mathrm{Pa}$, the average of this pressure gradient is $\sim 1.5 \times 10^{6} \mathrm{~Pa} \mathrm{~m}^{-1}$ through a boundary-layer thickness of $67.5 \mathrm{~mm}$. The earlier remark that there is a potentially large pressure gradient to provide for melt-water flow in the veins is substantiated.

The results also indicate that the thickness of the typical boundary layer should be small compared to the characteristic dimension of a subglacial water pocket. 
Therefore, the model, which assumes plane flow and is based on the analysis of a half space, should be applicable to the analysis of subglacial melting at water pockets.

Consider the case $Q<Q^{*}$. As noted, melt water from the boundary layer exits at the ice-water interface in a supercooled state. The proportion of melt water which refreezes will depend upon the thermodynamics of mixing with the water in the water pocket. For the idealized case where all the melt water refreezes, a steady-state regelation cycle exists between the ice and water pocket and no external heat input is required.

In the case of a small water pocket created by ice sliding over a bedrock bump, a regelation process operating between the stoss and lee sides of the bump has been established (Weertman, 1957, 1964; Kamb and LaChapelle, 1964). To the conventional regelation cycle must be added the ice-water pocket regelation cycle identified here.

The operation of the ice-water pocket regelation mechanism depends upon the ice in the boundary layer being warmed to near the steady-state temperature $\theta$ of the model as the ice moves over the water pocket. It is easy to establish that the required warming will occur unless there is an unusual combination of very rapid sliding and a water pocket of very small dimensions.

It is sufficient to consider a sinusoidal temperature input to the ice-water pocket surface with time period $t^{*}$. Let $y^{*}$ be the ice depth at which the temperature amplitude decays by factor $1-1 /$ e. Since the temperature amplitude decays as $\exp \left[-y\left(\pi / k t^{*}\right)^{\frac{1}{2}}\right]$ (Paterson, 1981, p. 187), where $k$ is the thermal diffusivity, we obtain

$$
y^{*}=\left(\boldsymbol{k} t^{*} / \pi\right)^{\frac{1}{2}} .
$$

It is appropriate to use values of $y^{*}$ from Table $I$ and solve for $t^{*}$ from Equation (22). Values of $t^{*}$ calculated in this manner represent the time required for a temperature wave with period $t^{*}$ to penetrate exponentially to depth $y^{*}$ These values of $t^{*}$ must then be compared with the actual time $\Delta t$ required for sliding ice to traverse one bed wavelength.

For example, using a small bed half-wavelength of $0.1 \mathrm{~m}$ and a large sliding velocity of $0.1 \mathrm{~m} \mathrm{~d}^{-1}$ gives $\Delta t=1 \mathrm{~d}$. Using the conservative value $y^{*}=95 \mathrm{~mm}$ from Table $I$, corresponding to $P_{0}-p_{\mathrm{W}}=10^{4} \mathrm{~Pa}$ and $\boldsymbol{B}=0$, gives $t^{*}=0.3 \mathrm{~d}$ from Equation (22). We conclude that, normally, the boundary layer will be heated early in the traverse of ice over a water pocket.

\section{CONCLUSIONS}

To the list of conclusions which can legitimately be made on the basis of the results, I shall add certain conclusions which contain an element of speculation.

A boundary layer of ice, probably considerably thinner than $0.1 \mathrm{~m}$, exists over water pockets at the base of glaciers, particularly glaciers with bedrock beds where ice-bed separation is apt to occur. Melting takes place within this boundary layer. Recycling of the boundary layer occurs to the extent that the ice-water-pocket-regelation mechanism operates. To the usual source of recrystallization must be added recrystallization in the boundary layer due to refreezing as part of the regelation process.

The water content is apt to be high in the boundary layer and crystal size small conforming to the results of Vallon and others (1976). The classical phenomenon of regelation sliding past small obstacles will be enhanced by the soft ice in the boundary layer (assuming that the water in the boundary layer is not immediately squeezed out after the ice re-contacts the bed) so that regelation sliding will be greater than predicted heretofore using properties of ordinary glacier ice.

The boundary layer, as defined here, does not appear to conform to the "sole" of the glacier, as suggested or defined by Barnes and Robin (1966). Thicknesses of the latter layer of clear ice appear to vary between 0.3 and $2 \mathrm{~m}$ or more (Robin, 1976). The boundary layer, however, does appear to agree with the observation of Carol (1947) of a basal layer of soft ice.

The creation of a boundary layer of soft ice requires only heat input and a normal bed pressure less than the nominal overburden pressure $\rho_{\mathrm{j}} g h$. Thus, the phenomenon described here should operate on the lee sides of the bed bumps for either a hard or soft bed, regardless of whether or not bed separation occurs. On the other hand, on the stoss side of bumps, heat input to the ice at the ice-bed interface will not result in interior melting of the ice because $\Delta P<0$. (On both lee and stoss sides, interior melting can result from internal energy dissipation due to strain-energy losses.)

Drainage of melt water through the vein system in the boundary layer is expected to occur for two reasons. Mechanical closure of the veins by large shear strain-rates should not occur, at least in the case where the boundary layer forms over a water pocket or region of ice-bed separation. Secondly, the pressure gradient in the vein system is potentially large because of the large gradient in the mean hydrostatic pressure through the boundary layer.

The phenomenon of the ice-water regelation cycle may explain ice crystals observed by LaChapelle (1968) growing into large water-filled cavities beneath thick ice where the conventional regelation mechanism does not operate. It may also be partially responsible for ice spicules observed growing into small cavities on the lee side of bedrock bumps (Kamb and LaChapelle, 1964). Alternatively, a source for these crystals and spicules could be the classical regelation cycle or an effect discussed by Lliboutry (1987, p. 163).

There is another effect which may be of importance. Any clast embedded in the boundary layer will tend to be pushed up into the ice wherever the ice-water pocket regelation mechanism (or an equivalent ice-water film mechanism) operates. This assumes that melt water generated in the boundary layer drains to the ice-water interface where it refreezes. The drainage flux is relative to the clast which remains in a fixed position relative to the ice (provided the downward velocity of the clast due to the gravitational force is neglected).

This effect has several additional implications. First, the debris-drag stress (Shoemaker, 1988) will be reduced because the spatially averaged speed of approach of a clast to the ice-bed interface, $v_{\mathrm{a}}$, will be reduced. Thus, relative to entrained clasts, there is an apparent freezing-on of ice even though the glacier is at the pressure-melting point throughout.

If net freezing-on relative to entrained clasts occurs, it is clear that the boundary layer will become a debris-rich layer. Debris will be incorporated in the glacier sole at water pockets, noting that water pockets on the lee side of bedrock bumps will be debris-rich. However, this mechanism cannot be the source of debris layers meters thick such as found at Byrd Station (Gow and others, 1979) and Camp Century (Herron and Langway, 1979). Thick debris layers are thought to originate by true freezing-on where subglacial conditions are not temperate.

The boundary layer is subject to extensional strain-rates in excess of the strain-rates in neighbouring ice. Because boundary-layer ice is either continuously recirculated through the regelation cycle, or melted, accumulated extensional strains in the boundary layer may not be so large that the ice fabric is drastically altered.

\section{ACKNOWLEDGEMENT}

I thank W.S.B. Paterson for helpful suggestions and for improving a very early version.

\section{REFERENCES}

Barnes, P. and G. de Q. Robin. 1966. Implications for glaciology. Nature, 210(5039), 882-883.

Carol, H. 1947. The formation of roches moutonneés. J. Glaciol., 1(2), 57-59.

Duval, P. 1977. The role of the water content on the creep rate of polycrystalline ice. International Association of Hydrological Sciences Publication 118 (Symposium at Grenoble 1975 - Isotopes and Impurities in Snow and Ice), 29-33.

Glen, J.W. 1955. The creep of polycrystalline ice. Proc. $R$. Soc. London. Ser. A, 228(1175), 519-538. 
Gow, A.J., S. Epstein, and W. Sheehy. 1979. On the origin of stratified debris in ice cores from the bottom of the Antarctic ice sheet. J. Glaciol., 23(89), 185-192.

Herron, S. and C.C. Langway, jr. 1979. The debris-laden ice at the bottom of the Greenland ice sheet. J. Glaciol., 23(89), 193-207.

Kamb, W.B. 1961. The thermodynamic theory of nonhydrostatically stressed solids. J. Geophys. Res., $66(1)$, 259-271.

Kamb, W.B. 1970. Sliding motion of glaciers: theory and observation. Rev. Geophys. Space Phys., 8(4), 673-728.

Kamb, W.B. and E. LaChapelle. 1964. Direct observation of the mechanism of glacier sliding over bedrock. $J$. Glaciol., 5(38), 159-172.

LaChapelle, E. 1968. Stress-generated ice crystals in a nearly isothermal two-phase system. $J$. Glaciol., $7(50)$, 183- 198.

Lliboutry, L. 1958. Contribution à la theorie du frottement du glacier sur son lit. C.R. Hebd. Séances Acad. Sci., Ser. $D, 247,318-320$.

Lliboutry, L. 1968. General theory of subglacial cavitation and sliding of temperate glaciers. $J$. Glaciol., 7(49), 21-58.

Lliboutry, L. 1971. Permeability, brine content, and temperature of temperate ice. J. Glaciol., 10(58), 15-29.

Lliboutry, L. 1976. Physical processes in temperate glaciers. J. Glaciol., 16(74), 151-158.

Lliboutry, L. 1978. Glissement d'un glacier sur un plan parsemé d'obstacles hémisphériques. Ann. Géophys., 34(2), 147-162.

Lliboutry, L. 1987. Very slow flows of solids: basis of modeling in geodynamics and glaciology. Dordrecht, etc., Martinus Nijhoff Publishers.

McLellan, A.G. 1966. A thermodynamical theory of systems under nonhydrostatic stresses. J. Geophys. Res., 71(18), $4341-4347$.

Nye, J.F. 1976. Water flow in glaciers: jökulhlaups, tunnels, and veins. J. Glaciol., 17(76), 181-207.
Nye, J.F, 1989. The geometry of water veins and nodes in polycrystalline ice. J. Glaciol., 35(119), 17-22.

Nye, J.F. and F.C. Frank. 1973. Hydrology of the intergranular veins in a temperate glacier. International Association of Scientific Hydrology Publication 95 (Symposium at Cambridge $1969-$ Hydrology of Glaciers), $157-161$.

Nye, J.F. and S. Mae. 1972. The effect of non-hydrostatic stress on intergranular water veins and lenses in ice. $J$. Glaciol., 11(61), 81-101.

Paterson, W.S.B. 1981. The physics of glaciers. Second edition. Oxford, etc., Pergamon Press.

Paterson, W.S.B. and J.C. Savage. 1970. Excess pressure observed in a water-filled cavity in Athabasca Glacier, Canada. J. Glaciol., 9(55), 103-107.

Robin, G. de Q. 1976. Is the basal ice of a temperate glacier at the pressure melting point? J. Glaciol., 16(74), 183-196.

Schneider, A. 1939. Unterwasser-Eiskristallbildung in untern Grindelwaldgletscher. Alpen, 17(10), 375-376.

Schneider, A. 1948. Submerged ice crystals in glaciers. $J$. Glaciol., I(4), 190-191.

Shoemaker, E.M. 1988. On the formulation of basal debris drag for the case of sparse debris. J. Glaciol., 34(118), 259-264.

Smith, G.D. and L.W. Morland. 1981. Viscous relations for the steady creep of polycrystalline ice. Cold Reg. Sci. Technol., 5(2), 141-150.

Vallon, M., J.-R. Petit, and B. Fabre. 1976. Study of an ice core to the bedrock in the accumulation zone of an Alpine glacier. J. Glaciol, 17(75), 13-28.

Vivian, R. and G. Bocquet. 1973. Subglacial cavitation phenomena under the Glacier d'Argentière, Mont Blanc, France. J. Glaciol., 12(66), 439-451.

Weertman, J. 1957. On the sliding of glaciers. J. Glaciol., 3(21), 33-38.

Weertman, J. 1964. The theory of glacier sliding. $J$. Glaciol., 5(39), 287-303. 